\title{
Coronary artery bypass graft surgery: socioeconomic inequalities in access and in 30 day mortality. A population-based study in Rome, Italy
}

Carla Ancona, Nera Agabiti, Francesco Forastiere, Massimo Arcà, Danilo Fusco, Salvatore Ferro, Carlo A Perucci

\begin{abstract}
Objectives-To evaluate whether coronary artery bypass graft (CABG) surgery is equally provided among different socioeconomic status (SES) groups in accordance with need. To estimate the association between SES and mortality occurring 30 days after CABG surgery.

Design-Individual socioeconomic index assigned with respect to the characteristics of the census tract of residence (level I = highest SES; level IV = lowest SES). Comparison of age adjusted hospital admission rates of ischaemic heart disease (IHD) and CABG surgery among four SES groups. Retrospective cohort study of all patients who underwent CABG surgery during 1996-97.
\end{abstract}

Setting-Rome (2 685890 inhabitants) and the seven cardiac surgery units in the city. Participants-All residents in Rome aged 35 years or more. A cohort of 1875 CABG patients aged 35 years or more.

Main outcome measures-Age adjusted hospitalisation rates for CABG and IHD and rate of CABG per 100 IHD hospitalisations by SES group, taking level I as the reference group. Odds ratios of 30 day mortality after CABG surgery, adjusted for age, gender, illness severity at admission, and type of hospital where CABG was performed.

Results-People in the lowest SES level experienced an excess in the age adjusted IHD hospitalisation rates compared with the highest SES level (an excess of 57\% among men, and of $94 \%$ among women), but the rate of CABG per 100 IHD hospitalisations was lower, among men, in the most socially disadvantaged level (8.9 CABG procedures per 100 IHD hospital admissions in level IV versus 14.1 in level I rate ratio $=0.63 ; 95 \%$ CI $0.44,0.89)$. The most socially disadvantaged SES group experienced a higher risk of 30 day mortality after CABG surgery $(8.1 \%)$ than those in the highest SES group (4.8\%); this excess in mortality was confirmed even when initial illness severity was taken into account (odds ratio $=2.89$; 95\% CI 1.44 , 5.80).

Conclusions-The universal coverage of the National Health Service in Italy does not guarantee equitable access to CABG surgery for IHD patients. Factors related to SES are likely to influence poor prognosis after CABG surgery.

(F Epidemiol Community Health 2000;54:930-935)
Although many studies have demonstrated that morbidity and mortality for various conditions are associated with socioeconomic status (SES), ${ }^{12}$ researchers have only recently begun to focus on two independent dimensions of equity: SES differences in access to treatment and SES differences in the outcome of medical interventions and surgical procedures. For various diseases, both people with low $\mathrm{SES}^{3}$ and ethnic minorities ${ }^{4}$ have been shown to have less equitable rates in the provision of health care than other sectors of the population. At the same time, survival after myocardial infarction or cancer has been found to be related to SES. ${ }^{5}{ }^{6}$

Several studies have shown a higher occurrence of ischaemic heart disease (IHD) among people with lower SES, ${ }^{7}$ even after controlling for cardiovascular risk factors. ${ }^{8}$ Coronary artery bypass graft (CABG) surgery is an effective treatment of IHD, ${ }^{9}$ and the provision of cardiac surgery can be used to test whether health care services are offered in an equitable fashion among patients who differ by SES. A recent study has suggested a strong effect of SES on access to specialised cardiac services in Canada, a country with an universal health care system. ${ }^{5}$ Moreover, access to CABG surgery has been found to be associated with SES and racial/ ethnic groups in the $\mathrm{UK}^{10}$ and in USA. ${ }^{41}$ At the same time, CABG surgery can be used to determine whether differences by SES exist in the effectiveness of treatment. Living in a poor community has been described as a significant and independent risk factor for mortality 36 months after CABG surgery. ${ }^{12}$ However, to the best of our knowledge, no studies have investigated the association between 30 day mortality after CABG surgery and SES.

To evaluate whether cardiovascular health care resources are equally provided among various SES groups in accordance with need, we investigated socioeconomic differences in the provision of CABG surgery in Rome (Italy). Moreover, we studied the association between SES and mortality occurring 30 days after CABG surgery.

\section{Methods}

DATA SOURCES AND SES

In Lazio, the region where Rome is located, hospital care (acute hospitalisation rate about 200 per 1000 inhabitants) ${ }^{13}$ is mostly covered by the National Health Service (NHS) through teaching and non-teaching hospitals. In Rome (2 685890 inhabitants) there are seven specialised hospitals with CABG units (three teaching hospitals, two non-teaching public hospitals, and two private-non-NHS-hospitals). 
Discharge data on all IHD and CABG patients, aged 35 years or more, residing in Rome and hospitalised in 1996-97, were obtained from the Lazio Region Hospital Information System (HIS).

Individual socioeconomic data on hospitalised people are not available. However, a method to assign a SES to each citizen in Rome has been developed based on information collected at the level of census tract of residence, the smallest territorial unit for which population data are available (average of 480 inhabitants in each census tract). ${ }^{14}$ In brief, this method included the development of a small area socioeconomic index for the inhabitants of each census tract, according to selected census variables, including level of education, occupation, dwelling ownership, family size, and people/room density. Four levels of SES were defined (that is, SES levels I, II, III, and IV); SES level I refers to the population with the highest SES. According to Municipal Registry data, in 1996 residents in the four categories were as follows: level I= 302 771; level $\mathrm{II}=514731$; level III= 459198 ; level IV= 279 059. Based on the home address, a SES level was assigned to each IHD and CABG patient.

PATIENTS

To study early mortality after CABG surgery, on the basis of data from the HIS, we identified a cohort of 1875 patients (aged 35 years or more) residing in Rome who underwent CABG surgery in any of the city's specialised hospitals with CABG units in the period 1996-97. Three people were excluded because information on the census tract of residence was missing; thus, 1872 patients were included in the analysis. The following individual information on patients was taken from the HIS database: gender, age, up to four diagnoses (ICD-9 codes) and up to four surgical procedures (ICD-9-CM codes) during hospital stay, dates of hospital admission and discharge. Vital status (dead or alive) 30 days after hospital discharge was obtained for each patient from the Municipal Registry of Rome, which collects demographic information on all the residents in the city. Death attributable to any cause was considered the study end point.

SEVERITY OF ILLNESS

To adjust mortality rates with respect to the severity of illness on admission, we identified variables known to be associated with 30 day mortality after CABG surgery:

(1) type of IHD (none, acute/subacute forms, angina pectoris, chronic forms, old myocardial infarction, acute myocardial infarction, mixed forms, defined when more than one type was reported);

(2) comorbidities, defined as chronic conditions apart from IHD that existed before hospital admission (other heart disease, diabetes, hypertension, chronic obstructive pulmonary disease (COPD), chronic renal disease, malignant neoplasm, peripheral arterial disease including cerebrovascular disease);
(3) heart surgery other than CABG and operations on arteries during the episode of care.

Comorbidities were defined according to the ICD-9 codes assigned by Deyo to the Charlson comorbidity index. ${ }^{15}$ Severity of illness did not include surgery related complications or death related conditions (for example, cardiac arrest).

ANALYSIS

We computed age standardised rates of hospitalisation for IHD (ICD-9 diagnosis codes 410-414) and CABG surgery (ICD-9-CM procedures code 36.1 ) by gender and SES level. We used all persons aged 35 years or more residing in Rome (1996) as the denominator population. All rates were directly standardised for age to the European standard population and expressed as the number of IHD hospital admissions and CABG procedures per 10000 inhabitants. Given the severity of IHD, hospital discharge data can provide a fair approximation of the occurrence within the population and may be considered as a proxy of the population's needs in terms of health care. We then analysed the age standardised ratio between the number of CABG operations and the number of IHD hospital admissions (CABG per 100 IHD) by SES level, separately for men and women.

We used rate ratios (RRs) to compare IHD hospital admission rates, CABG surgery rates, and CABG per 100 IHD among SES levels, using level I (highest SES) as the reference group. Confidence intervals (CI) were calculated at the $95 \%$ level of significance.

We evaluated the association between 30 day mortality and the four SES levels using logistic regression analysis (odds ratios (OR), and 95\% $\mathrm{CI})$. We adjusted for the following variables: age, gender, type of IHD, comorbidities, and other surgical procedures during the same admission (model 1: area under the receiver operating characteristic $($ ROC) curve $=0.789$ ). In addition, we evaluated a model including the type of hospital (teaching, non-teaching, and private) as a potential confounder (model 2: ROC $=0.799)$. All analyses were conducted using the statistical program STATA 5.0. ${ }^{16}$

\section{Results}

ACCESS TO CABG SURGERY AND SES

Table 1 shows the hospital admission rates (per 10 000) for IHD and CABG surgery by gender and SES level (level I = highest SES; level IV = lowest SES). In both genders, hospitalisation for IHD increased steadily with decreased SES. CABG hospitalisation rates among men were relatively constant among SES levels (RR $1.07,95 \%$ CI $0.84,1.35$ when comparing level IV to level I). For women with the lowest SES (level IV), an increase in the CABG hospitalisation rate was observed (RR 2.05, 95\% CI $1.22,3.45$ with respect to SES level I). However, the overall hospitalisation rate was lower among women than among men.

The rate of CABG per 100 IHD hospitalisations was lower among men with the lowest SES (RR 0.63, 95\% CI 0.44, 0.89 when comparing level IV to level I). Among women, no 
Table 1 Ischaemic heart disease (IHD) and coronary artery bypass graft (CABG) surgery annual hospital admission rates $\times 10$ 000 (age adjusted) by gender and socioeconomic status (SES), Rome 1996-1997. Level I = highest; level IV = lowest

\begin{tabular}{|c|c|c|c|c|c|c|c|c|c|c|c|c|}
\hline & \multicolumn{12}{|c|}{ SES level } \\
\hline & \multicolumn{3}{|l|}{$I$} & \multicolumn{3}{|l|}{$I I$} & \multicolumn{3}{|l|}{$I I I$} & \multicolumn{3}{|l|}{$I V$} \\
\hline & Number & Rate & $R R$ & Rate & $R R^{\star}$ & $95 \% C I$ & Rate & $R R^{\star}$ & $95 \% C I$ & Rate & $R R^{\star}$ & $95 \% C I$ \\
\hline \multicolumn{13}{|l|}{ Men } \\
\hline IHD & 2788 & 93.0 & 1.00 & 111.2 & 1.20 & $1.12,1.28$ & 121.7 & 1.31 & $1.23,1.40$ & 145.8 & 1.57 & $1.46,1.68$ \\
\hline CABG & 277 & 9.7 & 1.00 & 9.9 & 1.03 & $0.83,1.27$ & 11.0 & 1.14 & $0.92,1.41$ & 10.3 & 1.07 & $0.84,1.35$ \\
\hline $\begin{array}{l}\text { CABG per } 100 \mathrm{IHD} \\
\text { Women }\end{array}$ & & $14.1 \dagger$ & 1.00 & $10.3+$ & 0.73 & $0.52,1.03$ & $12.7 \dagger$ & 0.90 & $0.64,1.26$ & $8.9 \dagger$ & 0.63 & $0.44,0.89$ \\
\hline IHD & 1610 & 34.1 & 1.00 & 45.1 & 1.32 & $1.22,1.44$ & 53.4 & 1.57 & $1.44,1.70$ & 66.2 & 1.94 & $1.78,2.12$ \\
\hline CABG & 53 & 1.1 & 1.00 & 1.6 & 1.44 & $0.88,2.36$ & 1.4 & 1.30 & $0.78,2.17$ & 2.3 & 2.05 & $1.22,3.45$ \\
\hline CABG per $100 \mathrm{IHD}$ & & $4.3 \dagger$ & 1.00 & $8.8 \dagger$ & 2.08 & $0.78,5.55$ & $3.9 \dagger$ & 0.92 & $0.35,2.41$ & $4.9 \dagger$ & 1.15 & $0.45,2.97$ \\
\hline
\end{tabular}

${ }^{\star} \mathrm{RR}=$ rate ratios comparing occurrence of IHD and CABG hospital admissions in each SES level taking level I as the reference group. †Age standardised rate of CABG per 100 IHD hospital admissions.

statistically significant differences in this indicator with respect to SES level were observed.

PATIENTS CHARACTERISTICS AND MORTALITY

WITHIN 30 DAYS AFTER CABG

Most patients who had received a CABG surgery during the study were men $(83.4 \%)$; the proportion of women was higher in SES level IV compared with the other levels. Mean age was highest for those in SES level I (64.9 years versus 62.3 years for level IV). As expected, the percentage of patients treated in private hospitals was highest in level I (9.4\% compared with $0.6 \%$ for level IV) (table 2 ).

Patients with the lowest SES (level IV) showed a higher severity of illness upon hospital admission compared with patients with the highest SES (level I) (44.7\% of comorbidities versus $37.9 \%)$. Overall, hypertension and diabetes were the most frequently reported comorbidities $(18.3 \%$ and $13.5 \%$, respectively); diabetes was more common in SES level IV compared with level I (16.3\% versus 9.7\%) (table 3).

Table 2 Demographic characteristics and type of hospital of CABG surgery patients by socioeconomic status (SES), Rome 1996-1997. Level I = highest; level IV = lowest

\begin{tabular}{|c|c|c|c|c|c|c|c|c|c|c|}
\hline & \multicolumn{10}{|c|}{ SES level } \\
\hline & \multicolumn{2}{|l|}{$I$} & \multicolumn{2}{|l|}{$I I$} & \multicolumn{2}{|l|}{$I I I$} & \multicolumn{2}{|l|}{$I V$} & \multicolumn{2}{|l|}{ Total } \\
\hline & Number & $\%$ & Number & $\%$ & Number & $\%$ & Number & $\%$ & Number & $\%$ \\
\hline \multicolumn{11}{|l|}{ Sex } \\
\hline male & 277 & 83.9 & 505 & 83.1 & 499 & 86.3 & 281 & 78.9 & 1561 & 83.4 \\
\hline \multicolumn{11}{|l|}{$\operatorname{Age}(y)$} \\
\hline $35-49$ & 21 & 6.4 & 44 & 7.2 & 53 & 9.2 & 26 & 7.3 & 144 & 7.7 \\
\hline $50-69$ & 197 & 59.7 & 404 & 66.4 & 399 & 69.0 & 252 & 70.8 & 1252 & 66.9 \\
\hline$\geqslant 70$ & 112 & 33.9 & 160 & 26.3 & 126 & 21.8 & 78 & 21.9 & 475 & 25.4 \\
\hline mean age (SD) & \multicolumn{2}{|c|}{$64.9(8.9)$} & \multicolumn{2}{|c|}{$63.7(8.4)$} & \multicolumn{2}{|c|}{$62.5 \quad(8.5)$} & \multicolumn{2}{|c|}{$62.3(8.5)$} & \multicolumn{2}{|c|}{$63.3(8.6)$} \\
\hline \multicolumn{11}{|l|}{ Type of hospital } \\
\hline non-teaching & 59 & 17.9 & 164 & 27.0 & 170 & 29.4 & 135 & 37.9 & 528 & 28.2 \\
\hline private & 31 & 9.4 & 34 & 5.6 & 20.23 & 3.5 & 2 & 0.6 & 86 & 4.6 \\
\hline
\end{tabular}

Table 3 Clinical characteristics of CABG surgery patients by socioeconomic status (SES), Rome 1996-1997. Level I = highest; level IV = lowest

\begin{tabular}{|c|c|c|c|c|c|c|c|c|c|c|}
\hline & \multicolumn{10}{|l|}{ SES level } \\
\hline & \multicolumn{2}{|l|}{$I$} & \multicolumn{2}{|l|}{$I I$} & \multicolumn{2}{|l|}{$I I I$} & \multicolumn{2}{|l|}{$I V$} & \multicolumn{2}{|l|}{ Total } \\
\hline & Number & $\%$ & Number & $\%$ & Number & $\%$ & Number & $\%$ & Number & $\%$ \\
\hline \multicolumn{11}{|l|}{ Type of IHD } \\
\hline none & 3 & 0.9 & 6 & 1.0 & 8 & 1.4 & 2 & 0.6 & 20 & 1.0 \\
\hline acute/subacute forms & 37 & 11.2 & 66 & 10.9 & 69 & 11.9 & 46 & 12.9 & 30 & 11.6 \\
\hline angina pectoris & 7 & 2.1 & 0 & 0.0 & 10 & 1.7 & 8 & 2.2 & 20 & 1.3 \\
\hline chronic forms & 218 & 66.1 & 405 & 66.6 & 359 & 62.1 & 208 & 58.5 & 82 & 63.6 \\
\hline old myocardial infarction & 3 & 0.9 & 3 & 0.5 & 5 & 0.9 & 1 & 0.3 & 19 & 0.6 \\
\hline acute myocardial infarction & 8 & 2.4 & 15 & 2.5 & 18 & 3.1 & 4 & 1.1 & 21 & 2.4 \\
\hline mixed & 54 & 16.4 & 113 & 18.6 & 109 & 18.9 & 87 & 24.4 & 38 & 19.4 \\
\hline Comorbidities (at least one) & 125 & 37.9 & 261 & 42.9 & 264 & 45.7 & 159 & 44.7 & 62 & 43.2 \\
\hline other heart disease & 30 & 9.1 & 57 & 9.4 & 66 & 11.4 & 42 & 11.8 & 29 & 10.4 \\
\hline diabete & 32 & 9.7 & 84 & 13.8 & 78 & 13.5 & 58 & 16.3 & 32 & 13.5 \\
\hline hypertension & 49 & 14.8 & 114 & 18.7 & 113 & 19.5 & 67 & 18.8 & 37 & 18.3 \\
\hline COPD & 7 & 2.1 & 14 & 2.3 & 13 & 2.3 & 12 & 3.4 & 21 & 2.5 \\
\hline chronic renal disease & 3 & 0.9 & 12 & 2.0 & 8 & 1.4 & 1 & 0.3 & 20 & 1.3 \\
\hline malignant neoplasm & 1 & 0.3 & 1 & 0.2 & 0 & 0.0 & 1 & 0.3 & 19 & 0.2 \\
\hline peripheral arterial disease ${ }^{\star}$ & 22 & 6.7 & 43 & 7.1 & 50 & 8.6 & 17 & 4.8 & 26 & 7.0 \\
\hline Complications (at least one) & 17 & 5.1 & 33 & 5.4 & 39 & 6.7 & 23 & 6.5 & 25 & 6.0 \\
\hline local & 1 & 0.3 & 4 & 0.7 & 8 & 1.4 & 4 & 1.1 & 20 & 0.9 \\
\hline systemic & 16 & 4.8 & 31 & 5.1 & 33 & 5.7 & 21 & 5.9 & 24 & 5.4 \\
\hline Other operations on heart & 25 & 7.6 & 46 & 7.6 & 55 & 9.5 & 29 & 8.1 & 27 & 8.3 \\
\hline Operations on arteries & 4 & 1.2 & 16 & 2.6 & 17 & 2.9 & 2 & 0.6 & 21 & 2.1 \\
\hline
\end{tabular}

COPD $=$ chronic obstructive pulmonary disease. ${ }^{\star}$ Including cerebrovascular diseases. 
Table 4 Socioeconomic status and 30 day mortality among patients with CABG surgery, Rome 1996-1997

\begin{tabular}{llllllll}
\hline & Patients & Mortality (\%) & $\mathrm{OR}_{\text {crude }}$ & $\mathrm{OR}_{1}$ & $95 \% \mathrm{CI}$ & $\mathrm{OR}_{2}$ & $95 \% \mathrm{CI}$ \\
\hline \multicolumn{2}{l}{ Socioeconomic status } \\
I & 330 & 4.8 & 1.00 & 1.00 & & & \\
II & 608 & 7.4 & 1.57 & 1.91 & $0.99,3.67$ & 1.00 & \\
III & 578 & 4.1 & 0.85 & 1.07 & $0.52,2.19$ & 0.93 & $0.98,3.65$ \\
IV & 356 & 8.1 & 1.74 & 2.89 & $1.44,5.80$ & 2.45 & $1.21,4.96$
\end{tabular}

$\mathrm{OR}_{1}$, odds ratios, adjusted for age, gender, type of IHD, comorbidities, other operations on heart apart from $\mathrm{CABG}$, and operations on arteries. $\mathrm{OR}_{2}$, odds ratios, adjusted for age, gender, type of IHD, comorbidities, other operations on heart apart from CABG, operations on arteries, and type of hospital.

The overall crude mortality within 30 days after CABG surgery was $6.1 \%$, with excess among patients in SES level IV (8.1\%) compared with those in level I (4.8\%). After adjusting for gender, age, type of IHD, comorbidities, and other surgical procedures during the same admission, we found a statistically significant association between SES and 30 day mortality $(\mathrm{OR}=2.89 ; 95 \%$ CI $1.44,5.80$ when comparing level IV with level I) (table 4).

Variability in mortality was observed across hospitals: $5.0 \%$ for teaching hospitals, $9.5 \%$ for non-teaching hospitals, $2.3 \%$ for private hospitals; taking teaching hospitals as reference, and considering all the potential confounders included in model 1, non-teaching hospitals showed an excess in mortality risk $(\mathrm{OR}=2.10$, 95\% CI 1.32, 3.34). The association between SES level and mortality was confirmed, though slightly reduced $(\mathrm{OR}=2.45 ; 95 \%$ CI 1.21 , 4.96), when we included in the model, as potential confounder, the type of hospital, a variable possibly related to the quality of care, thus representing an intermediate factor in the association under study (model 2 in table 4).

The possible effect modification of gender, age, and type of hospital on the relation between SES and 30 day mortality was tested by forcing interaction terms in the logistic model. However, no significant contribution was observed.

\section{Discussion}

In Italy, as in the United Kingdom, the NHS theoretically provides universal coverage to any citizen in any part of the country without economic barriers to medical care. We found that, even though people with the lowest SES showed an excess in the age adjusted IHD hospitalisation rates compared with those with the highest SES (an excess of $57 \%$ among men and of $94 \%$ among women), the rate of CABG procedures per 100 IHD hospitalisations among men was lower in the most socially disadvantaged group. These data suggest that this sector of the population was receiving fewer operations than needed. Moreover, in our study, the most socially disadvantaged socioeconomic group experienced a higher risk of 30 day mortality after CABG surgery, even after taking into account differences in the initial severity of illness.

The medical decision to refer a patient for CABG surgery is complex. The physician's choice of whether a patient should be managed conservatively or surgically mainly depends on the patient's clinical characteristics (symptoms,
KEY POINTS

- Low social class people experience an excess in the age adjusted ischaemic heart disease (IHD) rates compared with people in the high social class level.

- Male patients in the low social class level receive coronary artery bypass graft (CABG) surgery less than those in the high social class level according to need.

- Socioeconomic status influence early mortality after CABG surgery, taking into account initial illness severity.

- Interventions to promote equal availability and effectiveness of CABG surgery for all citizens are recommended, even in countries with a NHS.

exercise tolerance, angiographic evidence of coronary artery disease, and myocardial functional status). ${ }^{17}$ Medium and high risk patients with stable coronary artery disease are eligible for CABG surgery. ${ }^{18}$ A SES related difference in clinical indications for CABG surgery is an obvious explanation for the observed differences in rates of utilisation of CABG procedures in our study. We did not obtain information on all IHD hospitalised patients' clinical characteristics to correctly estimate the need for surgery and the criteria used by clinicians. However, it is probable that IHD patients in the lower social class level had less favourable coronary profiles than people in the upper social class. In fact, disadvantaged patients tend to have more risk factors and more severe disease than those in the highest SES, ${ }^{7}{ }^{18} 19$ and an association between SES and IHD mortality was observed in Rome. ${ }^{14}$ This could produce a possible underestimation of the association between access to CABG surgery and SES. Furthermore, if CABG surgery is contraindicated for lower SES patients because of their more severe clinical conditions, this may imply a further discrimination, given that diagnostic and therapeutic measures for poor patients with IHD tend to be delayed compared with well off people. ${ }^{5}$

Another possible explanation of our results is that patients with low SES could have received percutaneous transuminal coronary angioplasty (PTCA), an alternative revascularisation procedure for IHD patients. PTCA is generally used for treatment of proximal and single vessel disease lesions and only recently it has been proposed as an effective alternative to CABG surgery in selected populations of patients with multivessel coronary artery disease. ${ }^{20}$ Therefore, patients who are referred for PTCA have better medical baseline characteristics than those undergoing CABG.$^{21}$ However, there is no evidence of the use of one revascularisation procedure for IHD patients compensating the lack of use of the other. ${ }^{22}$ Male age adjusted hospital admission rates for PTCA in Rome (1997; 580 hospitalisations) were not associated with SES level (level I 4.2 per 10 000; level IV 4.4 per 10 000).

We observed that women hospitalised for IHD undergo CABG surgery far less frequently than 
men. Such a sex bias in the delivery of CABG surgery has been previously reported, but the reasons of this evidence are still a matter of debate. ${ }^{23}{ }^{24}$ Sex related differences in natural history of disease, behavioural and life style risk factors, and benefits from surgery have been advocated to explain this difference. We failed to show differences by SES among women. We cannot exclude that this is attributable to the limited sample size of our study among women (given $\alpha=0.05$, we had a power of $80 \%$ to detect $R R \leqslant 0.59$ or $R R \geqslant 1.7$ ), but we can also presume that gender differences were so strong that inequalities in provision of CABG surgery could not be detected.

Sociodemographic factors may also interact with clinical characteristics to both directly and indirectly influence the physician's choice in diagnostic and therapeutic procedures. Hannan et $a l^{25}$ recently found that AfricanAmerican and Hispanic candidates were less likely to undergo CABG surgery than white patients despite the fact that CABG surgery was clinically judged to be appropriate and necessary. Moreover, given that those patients who smoke have poorer results after revascularisation procedures, ${ }^{26}$ many clinicians are reluctant to perform these procedures unless patients have stopped smoking. ${ }^{27}$ Finally, a strong inverse relation between income and rates of use and waiting times for invasive cardiac procedures has been recently found in a cohort of acute myocardial infarction patients in Canada, after controlling for patients' clinical characteristics. ${ }^{5}$

The decision of whether or not a procedure will be performed also depends on the patient's preferences for care, yet the issue of patient's preferences versus physician's judgement has received attention only recently. ${ }^{28}{ }^{29}$ In our study, no information was available to determine the importance of patient's awareness of their health status in explaining the observed differences among SES levels. It should also be pointed out that in Italy many surgeons belonging to the NHS also have private outpatient practices, though strict regulations to this regard have been issued. Thus, a patient who privately approaches a cardiologist employed by the NHS has a greater probability of receiving CABG surgery.

Mortality after CABG surgery is a function of the rate of occurrence of complications, and both mortality and complications have been shown to be closely related to the preoperative risk as predicted by different illness severity scores. ${ }^{30}$ Differences in the level of preoperative risk, including clinical symptoms, anatomic compromising of coronary vessels, adequacy of previous treatment, severity of comorbidities, immunological status, stress, depression, and social isolation could explain the finding that mortality after CABG surgery was highest among the people with the lowest SES. In fact, although people with low SES were significantly younger in our study, they had a higher prevalence of mixed forms of IHD (a presumable index of more severe disease), more comorbidities (diabetes, COPD), and more postoperative complications (table 3 ).
Differential standards of medical care and management, measured as percentage of medical injuries attributable to negligence, have been observed in New York. ${ }^{31}$ Egbert and Rothman have shown that African-Americans undergoing surgery are more likely to receive care from a physician in training than from a senior staff surgeon..$^{32}$ The possibility that people with the lowest SES level have the worst prognosis as a consequence of inadequate medical care during CABG hospitalisation is difficult to evaluate in our study. We lack detailed information on the process of care (that is, technical aspects of operations, preoperative and postoperative care programmes) and other possible factors influencing poor prognosis after CABG surgery such as the ability to adapt lifestyle (smoking, diet) and compliance to treatment.

The interpretation of our results is potentially limited by several factors. People were classified using a small area index: although this index strongly predicts socioeconomic inequalities in mortality in Rome,${ }^{14}$ it is still an aggregate index and individual data on various components of SES are lacking. However, aggregate indices of SES have been widely proposed in the $\mathrm{US}^{33}$ and in the $\mathrm{UK}^{34}$ as a valid mean of overcoming lack of individual information. The definition of the severity of illness was derived from the ICD-9 diagnosis and procedure codes, as assigned in the HIS database. Administrative data have been widely used to assess the effectiveness and quality of care provided by various services, especially in cardiac surgery. ${ }^{35}$ Although clinical datasets seem to be more accurate in detecting clinical and functional myocardial impairment, codebased measures have been shown to have good statistical performance in predicting inhospital deaths from CABG surgery. ${ }^{36}$ Moreover, we empirically attempted to distinguish comorbidities from complications and death related conditions, as suggested by other investigators, ${ }^{37}$ to improve the clinical validity of our model. We have no reason to suspect that the accuracy of coding may be biased toward a specific SES level.

Other studies have reported that access to a resource consuming procedure such as CABG surgery may be affected by SES. ${ }^{48}$ Inequalities in provision have been observed not only in the USA, where no NHS programme exists and where the patient's insurance status could thus influence access to care ${ }^{39}$ but also in Canada ${ }^{40}$ and the UK, ${ }^{41}$ countries with universal health coverage. Our study suggests that interventions to promote equal availability and effectiveness of CABG procedures will be crucial to reduce inequalities among social groups, even in countries with a NHS.

Funding: this work has been partly funded by the Italian National Health Service-Progetto "Efficacia ed equità dell'assistenza ospedaliera: pubblicizzazione e informazione ai cittadini" (Ministero della Sanità-Dipartimento della Programmazione "Programmi speciali" - Art 12, comma 2, lett b), del d lgs 502/92).

Conflicts of interst: none. 
1 Report of independent inquiry into inequalities in health. London: Stationary Office, 1998.

2 Stronks K, Mheen $\mathrm{H}$ van de, Mackenback JP. A higher prevalence of health problems in low-income groups: does it reflect relative deprivation? F Epidemiol Community Health 1998;52:548-57.

3 Khan KL, Pearson ML, Harrison EP, et al. Health care for Black and poor hospitalised Medicare patients. $7 A M A$ 1994;271:1169-74.

4 Goldberg KC, Hartz AJ, Jacobsen SJ, et al. Racial and community factors influencing coronary artery bypass graft 267:1473-7.

5 Alter DA, Naylor CD, Austin P, et al. Effects of socioeconomic status on access to invasive cardiac procedures and on mortality after acute myocardial infarction. N Engl F Med 1999;341:1359-67.

6 Rosso S, Faggiano F, Zanetti R, et al. Social class and cancer survival in Turin, Italy. $\mathcal{F}$ Epidemiol Community Health 1997;51:30-4.

7 Blane D, Hart CL, Davey Smith G, et al. Association of cardiovascular disease risk factors with socioeconomic cardiovascular disease risk factors with socioeconomic
position during childhood and during adulthood. BMf position during chil.

8 Gliksman MD, Kawachi I, Hunter D, et al. Childhood socioeconomic status and risk of cardiovascular disease in middle aged US women: a prospective study. 7 Epidemiol Community Health 1995;49:10-15.

9 Yusuf S, Zucker D, Peduzzi P, et al. Effect of coronary artery bypass graft surgery on survival: overview of 10 -year results from randomized trials by the Coronary Artery Bypass Surgery Trialist Collaboration. Lancet 1994;344: Bypass $563-70$.

10 Morrison C, Woodward M, Leslie W, et al. Effect of socioeconomic group on incidence of, management of, and survival after myocardial infarction and coronary death: analysis of community coronary event register. BMF 1997; 314:541-6.

11 Ford E, Cooper RS. Racial/ethnic differences in health care utilization of cardiovascular procedures: a review of the evidence. Health Serv Res 1995;30:237-52.

12 Boscarino JA, Chang J. Survival after coronary artery bypass graft surgery and community socioeconomic status.
Clinical and research implications. Med Care 1999;37:210Clin

13 Arcà M, Cesaroni G, De Pascali V, et al. RAD 1997. Rapporto sull'attività di assistenza ospedaliera nel Lazio. Progetto Salute 1998;41:17-46.

14 Michelozzi P, Perucci CA, Forastiere F, et al. Inequality in health: increasing socioeconomic differentials in mortality in Rome, 1990-95. F Epidemiol Community Health 1999;53: 687-93.

15 Deyo RA, Cherkin DC, Ciol MA. Adapting a clinical comorbidity index for use with ICD-9-CM administrative databases. F Clin Epidemiol 1992;45:613-19.

16 STATA for Windows release 5. College station,TX: Stata Press, 1997.

17 Leape LL, Hilborne LH, Park RE. The appropriateness of use of coronary artery bypass graft surgery in New York State. $7 A M A$ 1993;269:753-60.

18 Yusuf S, Zucker D, Peduzzi P, et al. Effect of coronary artery bypass graft surgery on survival: overview of 10-year results from randomized trials by the Coroanry Artery Bypass Graft Surgery Trialists Collaboration. Lancet 1994;344 563-70.

19 Wenneker MB, Epstein AM. Racial inequalities in the use of procedures for patients with ischemic heart disease in Massachusetts. FAMA 1989;261:253-7.
20 Carriè D, Elbaz M, Puel J, et al. Five-year outcome after coronary angioplasty versus bypass surgery in multivessel coronary artery disease. Results from the French monocentric study. Circulation 1997:96 (suppl II):II-1-6.

21 Vacek JL, Rosamond TL, Stites HW, et al. Comparison of PTCA versus CABG for multivessel coronary artery disease. Am f Cardiol 1992;69:592-7.

22 Black N, Langham S, Petticrew M. Coronary revascularization: why do rates vary geographically in the UK. $\mathcal{F}$ Epidemiol Community Health 1995;49:408-12.

23 Petticrew M, McKee M, Jones J. Coronary artery surgery: are women discriminated against?. BMF 1993;306:1164-6.

24 Tobin JN, Wassrtheil-Smoller S, Wexler JP, et al. Sex bias in considering corona

25 Hannan EL, Van Ryn M, Burke J, et al. Access to coronary artery bypass surgery by race/ethnicity and gender among patients who are appropriate for surgery. Med Care 1999;37:68-77.

26 Cameron AA, Davis KB, Rogers WJ. Recurrence of angina after coronary artery bypass surgery: predictors and prognosis (CASS registry). Coronary Artery Surgery prognosis (CASS registry). Coronary
Study. F Am Coll Cardiol 1995;26:895-9.

27 Underwood MJ, Bailey JS. Should smokers be offered coronary bypass surgery? BMf 1993;306:1047-8.

28 Sedlis SP, Fisher VJ, Tice D, et al. Racial differences in perormance of invasive cardiac procedures in a Department of Veterans Affairs Medical Center. $\mathcal{f}$ Clin Epidemiol 997;50:899-901.

29 Maynard C, Fisher LD, Passamani ER, et al. Blacks in the coronary artery surgery study (CASS): race and clinical decision making. Am f Public Health 1986;76:1446-8.

30 Ghali 1998, Hall RE, Ash AS, et al. Evaluation of complication rates after coronary artery bypass surgery using administrative data. Methods Inf Med 1998;37:192-200.

31 Burstin HR, Lipsitzz SR, Brennan TA. Socioeconomic status and risk for substantial medical care. $\mathcal{F} A M A$ 1992;268: $2383-7$

32 Egbert LD, Rothman IL. Relation between race and economic status of patients and who performs their surgery. N Engl Med 1977;297:90-1.

33 Krieger N. Overcoming the absence of socioeconomic data in medical records: validation and application of a census-

34 Danesh J, Gault S, Semmence J, et al. Postcodes as useful markers of social class: population based study in 26.000 British households. BMf 1999;318:843-5.

35 Iezzoni LI. Assessing quality using administrative data. Ann Intern Med 1997;127:666-74.

36 Iezzoni LI, Ash AS, Shwartz M, et al. Predicting in-hospital deaths from coronary artery bypass graft surgery. Do different severity measures give different predictions? Med Care 1998;36:28-39.

37 Elixhauer A, Steiner C, Harris R, et al. Comorbidity measures for use with administrative data. Med Care 1998; 36:8-27.

38 Hannan EL, Kilburn H, O'Donnell J, et al. Interracial access to selected cardiac procedures for patients hospitalised with coronary artery disease in New York State. Med Care 1991; 29:430-41.

39 Hadley J, Steinberg EP, Feder J. Comparison of uninsured and privately insured hospital patients. Condition on admission, resource use, and outcome. $\mathscr{F} A M A$ 1991;265: 374-9.

40 Anderson GM, Grumbach K, Luft HS, et al. Use of coronary artery bypass surgery in the United States and Canad. Influence of age and income. $\mathcal{F} A M A$ 1993;269:1661-6.

41 Mayeed FA, Chaturvedi N, Reading R, et al. Monitoring and promoting equity in primary and secondary care. $B M$ 1994;308:1426-9. 